\title{
ANÁLISE MORFOLÓGICA DENTÁRIA DOS TRAÇOS NÃO-MÉTRICOS DE UMA AMOSTRA REGIONAL
}

\author{
Renata Aparecida Pauli de Oliveira Ricco, Élcio Mario Faria Junior, Aisy Tetila Dias \\ Universidade do Oeste Paulista - UNOESTE, Curso de Odontologia, Presidente Prudente, SP. e-mail: renataricco@unoeste.br
}

\section{RESUMO}

Os traços não-métricos são um dos principais focos de observação para análise da variação humana e sua relação com histórico das populações. O objetivo do trabalho foi realizar uma análise morfológica dentária dos Traços não-métricos de uma amostra regional. Foram analisados modelos de gesso de 50 indivíduos do sexo masculino e 50 do sexo feminino. Avaliou-se a presença de seis Traços morfológicos dentários: Incisivo em forma de pá (incisivo central superior), tubérculo de Carabelli (primeiro molar superior), quinta cúspide ou tubérculo distal acessório (primeiro molar superior), ausência da cúspide disto-palatina ou da quarta cúspide (segundo molar superior), sexta cúspide ou tubérculo sexto (primeiro molar inferior), ausência de cúspide disto-vestibular ou molar inferior tetra-cuspidado (segundo molar inferior). Os Traços com maior ocorrência foram $M_{2}$ (ausência de cúspide DV) em $92 \%$ e o de menor, $M_{1}$ (6a cúspide) em $4 \%$. Houve ausência ou diminuição dos traços não-métricos estudados.

Palavras-chave: dentição permanente, odontologia, antropologia, grupos étnicos, odontologia forense.

\section{DENTAL MORPHOLOGICAL ANALYSIS OF NON-METRICAL TRAITS CONCERNING A REGIONAL SAMPLE}

\section{ABSTRACT}

The non-metric traits are a major focus of observation to analysis of human variation and its relationship to historic of populations. The objective was carried out a Dental Morphological analysis of non-metrical traits concerning a regional sample. Plaster models were analyzed of 50 males and 50 females individuals. It was evaluated the presence of 6 dental morphological traits: spatula-shaped incisors (maxillary central incisor), Carabelli tubercle (first superior molar), fifth cusp or accessory distal tubercle (first superior molar), distalpalatal cusp or fourth cusp (second superior molar) absence, sixth cusp or sixth tubercle (lower first molar), no distobuccal cusp or tetra-cuspidate lower molar (lower second molar). The trait with higher percentage of occurrence were $M_{2}$ (no DV cusp) in $92 \%$ and, the minor occurrence, $M_{1}\left(6^{\text {th }}\right.$ Cusp) at $4 \%$. There was absence or reduction of traits non-metric studied.

Keywords: permanent dentition, dentistry, anthropology, ethnic groups, forensic dentistry.

\section{INTRODUÇÃO}

A evolução da espécie humana, em seu processo de adaptação, tem apresentado algumas variações biológicas ou características como: cor da pele, forma do cabelo, cor dos olhos etc. Estas variações biológicas, transmitidas por herança, juntamente com as diferenças culturais, recebem o conceito atual de etnia representada por três grandes grupos étnicos: caucasóides (brancos), negróides (negros) e mongolóides (amarelos) $^{1,2}$.

$\mathrm{Na}$ morfologia dental, algumas características dentárias, podem servir para a estimativa da idade, do sexo e etnia ${ }^{3}$.

Os elementos dentais podem ser estudados quanto a aspectos métricos e nãométricos. "Métricas" são características medidas diretamente, e que podem ser traduzidas em valores numéricos. Por outro lado, "nãométricos" implica aspectos registrados visualmente, em termos de ausência, presença, grau de desenvolvimento ou forma ${ }^{2,4}$.

Dentre as características dentárias determinantes da etnia incluem: o tamanho, a forma dos dentes, os incisivos em forma de pá e o Tubérculo de Carabelli, ${ }^{4,5,6}$. Segundo alguns autores, a característica dentária mais útil na determinação da etnia são os incisivos em forma de pá, encontrados em quase todos os Asiáticos ou Mongolóides, e em menos de $10 \%$ dos Caucasianos e Negróides ${ }^{6,7}$. Ainda no que diz respeito aos grupos étnicos, verifica-se que nos negros africanos, os dentes mostram-se, em 
geral, mais volumosos que nos indivíduos brancos ${ }^{8,9}$.

As principais características étnicas encontram-se presentes, especialmente, nos molares, através dos quais é possível diferenciar as etnias ${ }^{10}$. Na dentição dos primatas e fósseis é bastante incomum encontrar molares inferiores com menos de cinco cúspides. $O$ dente focal para o estudo da cúspide cinco é o segundo molar inferior, face á maior variabilidade interpopulacional registrada. A ausência da quinta cúspide no segundo molar inferior ocorre mais frequente que no primeiro ${ }^{11}$. Tal características permite á investigações no arco do indivíduo vivo, usando as mesmas técnicas de fósseis ${ }^{1,12}$.

As pesquisas mostram que os brasileiros apresentam alto grau da ancestralidade europeia do lado paterno, enquanto as ancestralidades ameríndias e africanas predominam do lado materno ${ }^{13}$.

Efetivamente, o Brasil talvez seja um dos países onde aconteceu a maior miscigenação entre os três grandes grupos étnicos geográficos. Ocorre ainda que, com sua dimensão continental, suas populações sofreram variadas influências genéticas, em diferentes regiões. No Norte e Nordeste houve considerável miscigenação trihíbrida, com a participação de diferentes populações de brancos, índios e negros. Já no Sudeste preponderou a miscigenação bi-híbrida de brancos com negros. No extremo Sul (PR, SC e RS) houve preponderância caucasiana, embora a influencia negróide e uns poucos genes índios não possam ser desprezados ${ }^{14}$.

Este trabalho através do reconhecimento de Traços dentário de uma população se mostra relevante com respeito a marcadores inter $\mathrm{e}$ intragrupos que permitirá futuras comparações entre populações humanas, esclarecendo mais aspectos sobre evolução, migrações, miscigenação, apresentando também grande colaboração para fins periciais em contexto forense. Portanto, o objetivo deste estudo foi realizar uma análise morfológica dentária dos Traços não-métricos de uma amostra regional.

\section{MATERIAL E MÉTODOS}

A metodologia constou de um estudo dos modelos de gesso das arcadas dentárias de 100 indivíduos (50 homens e 50 mulheres), em tratamento, após assinatura do termo de consentimento livre e esclarecido. 0 projeto foi avaliado e aprovado pelo Comitê de Ética em Pesquisas (núm. CAAE: 22372714.0.0000.5515).

Foram submetidos à avaliação da ocorrência de seis Traços não métricos. As amostras estudadas foram obtidas do acervo de documentação ortodôntica de um centro de Ortodontia em Presidente Prudente, SP, com os seguintes critérios de inclusão: modelos com dentição permanente de pacientes com faixa etária de 16 a 26 anos; habitantes de Presidente Prudente; pele branca e ausência de anomalia evidente.

As análises das amostras foram obtidas através dos traços registrados pelo método nominal, dicotomizado, identificados como "ausente" ou "presente". Traços observados:

Incisivo em forma de pá: o dente alvo avaliado é o incisivo central superior (Figura 1).

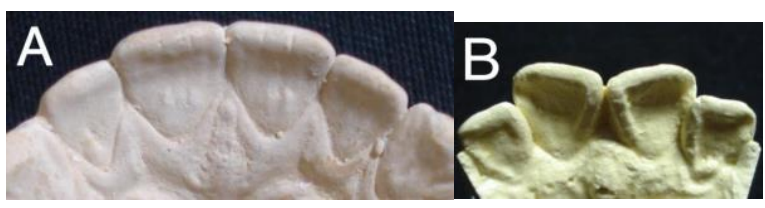

Figura 1. Incisivo em forma de pá. A: ausente; B: presente. Fonte: Tinoco ${ }^{5}$.

Tubérculo de Carabelli: o dente alvo foi o primeiro molar superior (Figura 2).

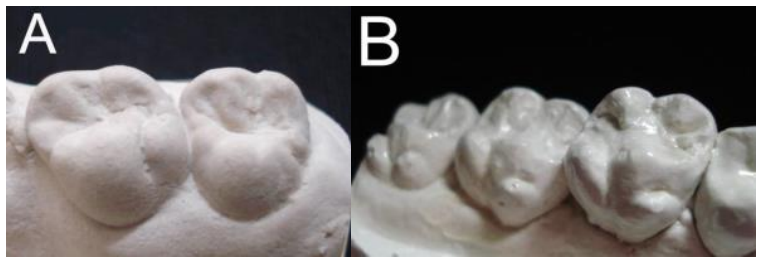

Figura 2. A: Primeiro molar superior sem tubérculo de Carabelli. B: Primeiro molar superior com marcante expressão do tubérculo de Carabelli. Fonte: Tinoco ${ }^{5}$.

Quinta cúspide: o dente alvo pesquisado foi o primeiro molar superior (Figura 3). 


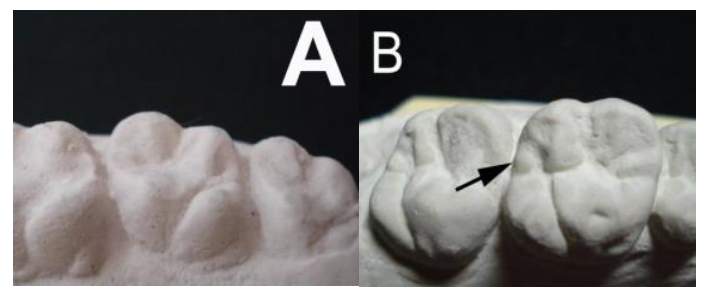

Figura 3. A: Primeiro molar superior sem nenhuma expressão de $5^{\text {a }}$ cúspide; B: Primeiro molar superior com presença de $5^{\text {a }}$ cúspide, assinalada pela seta. Fonte: Tinoco ${ }^{5}$.

Ausência da cúspide disto-palatina: o dente alvo foi o segundo molar superior (Figura 4).

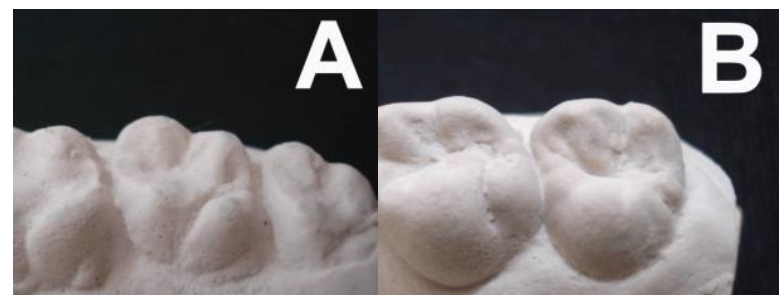

Figura 4. A: Segundo molar superior (no centro) com $4^{\text {a }}$ cúspide; B: Segundo molar superior (à direita) sem $4^{\text {a }}$ cúspide (molar superior tricuspidado). Fonte: Tinoco ${ }^{5}$.

Sexta cúspide ou tubérculo sexto: o dente alvo foi o primeiro molar inferior (Figura 5).

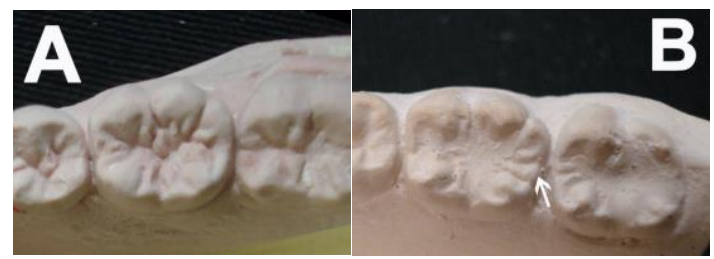

Figura 5. A: Primeiro molar inferior sem 6a cúspide; B: Primeiro molar inferior com presença de $6^{a}$ cúspide, assinalada pela seta. Fonte: Tinoco ${ }^{5}$.

Ausência de cúspide disto-vestibular: 0 dente focal para exame é o segundo molar inferior Figura 6).

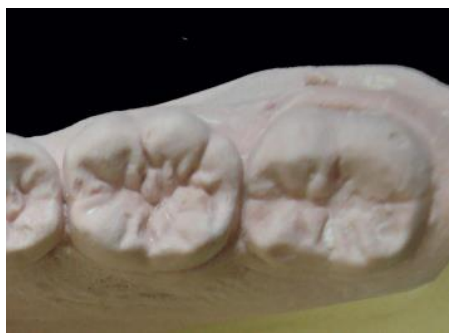

Figura 6. Primeiro molar inferior mostrando as cinco cúspides presentes, e segundo molar com ausência da $5^{a}$ cúspide, resultando em um molar inferior tetra-cuspidado.

Para análise utilizou-se o teste para duas proporções, que permite verificar, ao nível de significância $\alpha=0,05$, se as proporções de determinada característica observada em duas amostras aleatórias são diferentes.

\section{RESULTADOS}

Foram examinados modelos em gesso de 100 (cem) indivíduos entre 16 e 26 anos, distribuídos entre sexo e faixas etárias conforme explicitado pela tabela 1 .

Tabela 1. Distribuição da amostra de acordo com sexo e faixa etária.

\begin{tabular}{l|c|c|c|c|c|c}
\hline \multirow{2}{*}{ Idade } & \multicolumn{2}{|c|}{ Feminino } & \multicolumn{2}{r|}{ Masculino } & \multicolumn{2}{c}{ Total } \\
\cline { 2 - 7 } & $\mathbf{n}$ & $\%$ & $\mathbf{n}$ & $\%$ & $\mathbf{n}$ & $\%$ \\
\hline 16 a 20 & 31 & 62 & 23 & 46 & 54 & 54 \\
21 a 26 & 19 & 38 & 27 & 54 & 46 & 46 \\
\hline Total & 50 & 100 & 50 & 100 & 100 & 100 \\
\hline
\end{tabular}

Na Tabela 2 nota-se que, para os Traços "Hipocone ausente ou segundo molar superior tricuspidado" e "Hipocônulido ausente ou segundo molar inferior tetracuspidado", a proporção de incidência no sexo feminino apresentou diferença estatisticamente significante em relação à incidência no sexo masculino. Quanto aos demais Traços, não existem evidências estatísticas para afirmar que as proporções sejam diferentes. 
Tabela 2. Número e proporção de dentes observados e valor de $p$ referente ao teste para duas proporções, segundo traço e sexo.

\begin{tabular}{lcccccccc}
\hline \multirow{2}{*}{ Traço } & \multicolumn{2}{c}{ Feminino } & \multicolumn{2}{c}{ Masculino } & \multicolumn{2}{c}{ Total } & \multirow{2}{*}{$\boldsymbol{p}$} \\
\cline { 2 - 7 } & Freq. Abs. & $\%$ & Freq. Abs. & $\%$ & Freq. Abs. & $\%$ & \\
\hline Forma de pá $\left(\mathrm{I}^{1}\right)$ & 22 & 22 & 24 & 24 & 46 & 23 & 0,737 \\
\hline Carabelli $\left(\mathrm{M}^{1}\right)$ & 56 & 56 & 55 & 55 & 111 & 56 & 0,887 \\
\hline 5a cúspide $\left(\mathrm{M}^{1}\right)$ & 8 & 8 & 7 & 7 & 15 & 8 & 0,788 \\
\hline Hipocone ausente $\left(\mathrm{M}^{2}\right)$ & 66 & 66 & 48 & 48 & 114 & 57 & 0,009 \\
\hline 6a cúspide $\left(\mathrm{M}_{1}\right)$ & 3 & 3 & 5 & 5 & 8 & 4 & 0,470 \\
\hline Hipocônulido ausente $\left(\mathrm{M}_{2}\right)$ & 96 & 96 & 88 & 88 & 184 & 92 & 0,035 \\
\hline
\end{tabular}

Considerando as frequências citadas por Tinoco $^{5}$ e Irish $^{18}$, fatos históricos registrados neste país e na região Sudeste a marcante influência dos complexos caucasóide e subsaariano sob o ponto de vista antropológico dental foram encontrados na amostra analisada, resumidos na Tabela 3.

Tabela 3. Proporção de dentes observados, segundo traço e região geográfica.

\begin{tabular}{|c|c|c|c|c|}
\hline Traço & $\begin{array}{c}\text { Europa* } \\
\%\end{array}$ & $\begin{array}{c}\text { África* } \\
\%\end{array}$ & $\begin{array}{c}\text { Rio de Janeiro ** } \\
\%\end{array}$ & $\begin{array}{c}\text { P. Prudente*** } \\
\%\end{array}$ \\
\hline Forma de pá $\left(I^{1}\right)$ & 2,60 & 5,30 & 14,29 & 23,00 \\
\hline Carabelli ( $\left.\mathrm{M}^{1}\right)$ & 24,00 & 16,40 & 19,35 & 56,00 \\
\hline 5a cúspide $\left(\mathrm{M}^{1}\right)$ & 17,20 & 32,80 & 24,78 & 8,00 \\
\hline Hipocone ausente $\left(\mathrm{M}^{2}\right)$ & 22,30 & 9,70 & 58,33 & 57,00 \\
\hline 6a cúspide $\left(M_{1}\right)$ & 11,50 & 16,60 & 14,95 & 4,00 \\
\hline Hipocônulido ausente $\left(\mathrm{M}_{2}\right)$ & 77,00 & 24,10 & 83,04 & 92,00 \\
\hline
\end{tabular}

Com base na Tabela 3, sugere-se que, para os Traços "Carabelli", "5 5 cúspide" e "6a cúspide", as proporções referentes à amostra da Europa são as mais próximas comparadas as de Presidente Prudente, ao passo que, para os Traços "Forma de pá", "Hipocone ausente" e "Hipocônulido ausente", as proporções referentes à amostra do Rio de Janeiro são as mais próximas com as de Presidente Prudente.

\section{DISCUSSÃO}

O estudo da morfologia dentária através dos caracteres não-métricos é uma das formas hoje reconhecidas de estimativa de proximidade e diferenciação genética entre populações tanto diacronicamente como sincronicamente ${ }^{7}$.

Em primeiro lugar, pelo fato das estruturas dentárias representarem os mais duros e resistentes tecidos do corpo humano, resistindo á putrefação, ao calor, aos traumatismos e á ação de certos agentes químicos. Em segundo lugar, parece haver uma genética tanto no desenvolvimento dentário como na expressão de caracteres. Um terceiro fator é a facilidade de observação tanto em populações vivas quanto arqueológicas ${ }^{8,9}$.

A pesquisa de variação biológica humana possui importantes implicações sociais, biomédicas e forenses ${ }^{15}$.

De acordo com os resultados obtidos da amostra estudada, os Traços apresentaram praticamente $100 \%$ de ocorrências bilaterais. Seguramente estudos futuros poderão avaliar apenas um hemi-arco dentário.

Segundo um estudo genético autossômico de 2010, a herança europeia seria dominante entre os brasileiros, por volta de $80 \%$ do patrimônio genético da população, justificando os resultados que sugerem para os 
Traços "Carabelli", "5a cúspide" e "6 6 cúspide" as proporções mais próximas à amostra da Europa com as da amostra de Presidente Prudente.

Este estudo sugere que com a evolução humana, houve ausência ou redução de alguns traços não métrico, com tendência de simplificação da anatomia dental, necessitando mais estudos para constatar essas observações.

\section{CONCLUSÃO}

Concluimos que os traços com maiores de ocorrências foram $M_{2}$ (ausência de cúspide DV) em $92 \%$, seguido da $M^{2}$ (ausência de cúspide $D L$ ) em $57 \%$ e $M_{1}$ (tubérculo de Carabelli) em $56 \%$. Os Traços de menores ocorrências foram $M_{1}$ (6a cúspide) em $4 \%$, seguido pelo $M^{1}$ (5a cúspide) em $8 \%$ e de $I^{1}$ (em pá) 23\%. Houve ausência ou diminuição do traço não-métrico estudado. Os Traços não-métricos dentários apresentaram um alto valor identificativo, sendo importante sua aplicação complementar na odontologia forense.

\section{CONFLITO DE INTERESSE}

Os autores declaram não haver qualquer potencial de conflito de interesse que possa interferir na imparcialidade deste trabalho científico.

\section{REFERÊNCIAS}

1. Hillson S. Dental anthropology. Cambridge: Cambridge University Press; 2002.

2. Krenzer U. Antropologia dental: introdução, morfologia dental. In: Krenzer U. Compêndio de métodos antropológico forenses para la reconstruccíon del perfil osteo-biologico. 1.ed. Guatemala: Centro de Analisis Forenses y Ciencias Aplicadas; 2006. p.1-2.

3. Peres AS, Silva RA. Odontologia legal: manual resumo: compêndio de grandes obras de odontologia legal e medicina legal. 2004. p.95.

4. Etchegoyen CAS. Antropologia física - arcadas dentárias. [Dissertação]. Piracicaba: Faculdade de Odontologia de Piracicaba da Universidade Estadual de Campinas; 2011.

5. Tinoco RLR. Antropologia dental: traços nãométricos de uma amostra Brasileira. [Dissertação]. Piracicaba: Faculdade de Odontologia de Piracicaba da Universidade Estadual de Campinas; 2010.

6. Silva RF, Chaves P, Paranhos LR, Lenza MA, Daruge Jr E. Utilização de documentação ortodôntica na identificação humana. Dent Press J Orthodon. 2011;16(2):52-7.
http://dx.doi.org/10.1590/S2176-

94512011000200007

7. Scott GR. Dental morphology. In: Scott GR. Biological anthropology of the human skeleton. 2.ed. New York: John Wiley \& Sons; 2008. p.264-98. DOI: http://dx.doi.org/10.1002/9780470245842.ch9

8. Della Serra, O. Observações morfológicas sobre os dentes jugais humanos. Rev Assoc Paul Cir Dent. 1951; 37-45.

9. Irish JD. Characteristic high-and low-frequency dental traits in sub-saharan African populations Am J Phys Anthropol. 1997;102:455-67. DOI:

http://dx.doi.org/10.1002/(SICI)1096-

8644(199704)102:4<455::AID-AJPA3>3.0.CO;2-R

10. Edgar $\mathrm{HJH}$, Hunley KL. Race reconciled? How biological anthropologists view human variation. Am J Phys Anthropol. 2009;139:1-4. DOI: http://dx.doi.org/10.1002/ajpa.20995

11. Cunha C. Caracterização da morfologia dentária no neolítico final-calcolítico. Fundamentação para o mapeamento morfologia das populações locais na préhistoria recente. Apontam Arqueol Patrim. 2012;8:4955.

12. Santos AB. Estudos Arqueológicos a serviço de contextos criminais: Arqueologia forense. [Tese]. Universidade Federal de Sergipe UFS; 2011.

13. Petrucelli JL, Saboia AL. Características ÉtnicoRaciais da População. 2.ed. Rio de Janeiro: IBGE; 2013. 9-12.

14. Hernández J, Villavicencio J, Arce E, Moreno F. Talóncuspídeo: reporte de cinco casos. Rev Facultad Odontol Univ Antioquia. 2010;21(2):208-17.

15. Peres AS. Identificação de cadáveres através da arcada dentária. Rev Odontol Araçatuba. 2006;27(1):25-7.

16. Ferreira MA, Hespanhol LC, Oliveira Capote TS, Gonçalves MA, Campos JADB. Presença e morfologia do tubérculo molar de acordo com a dentição, hemiarco e sexo. Int J Morphol. 2010;28(1):121-5. DOI: http://dx.doi.org/10.4067/s0717-

95022010000100017

17. Sadatullah S, Odusanya SA, Mustafa A, Razak PA, Wahab MA, Meer Z. A prevalência de quinta cúspide (cúspide de Carabelli) nos molares superiores em escolares do ensino da Arábia Saudita. Int J Morphol. 2012;30(2):757-60.

DOI:

http://dx.doi.org/10.4067/S071795022012000200066

18. Irish JD, Guatelli-Steinberg D. Ancient teeth and modern human origins: an expanded comparison of African Plio-Pleistocene and recent world dental samples. J Human Evolut. 2003;45:113-144.

Recebido para publicação em 20/08/2015

Revisado em 26/08/2015

Aceito em 14/09/2015 